\title{
Jefferson. Stromal Caveolin-1 Levels Predict Early Ductal Carcinoma in Situ Progression to Invasive Breast Carcinoma
}

\section{Witkiewicz AK, Dasgupta A, Schwartz GF, Nguyen KHH, Rui HR, Lisanti MP}

Jefferson University, Philadelphia, PA; Kimmel Cancer Center, Philadelphia, PA.

\section{Introduction}

With the increased use of screening mammography, the incidence of ductal carcinoma in situ (DCIS) has increased substantially and it currently accounts for $20-30 \%$ of newly diagnosed breas carcinomas in United States. Breast conserving therapy with or without radiotherapy is the accepted

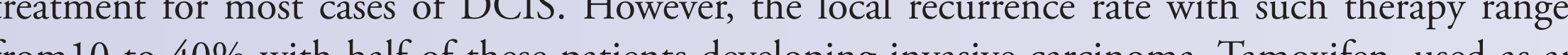
J jurat $40 \%$ wich he recurrence rates are conflicting.

The current classification for DCIS based on nuclear grade, architectural differentiation and presence of necrosis, does not adequately predict the likelihood of recurrence after breas progression and potential targets for therapy.

Recently, we showed that an absence of stromal (caveolin 1) Cav-1 expression in human breas cancers is a powerful single independent predictor of early disease recurrence, metastasis and poor clinical outcome. However, it remains unknown whether stromal Cav-1 levels play any role in the progression of DCIS. The aim of this study was to evaluate the stromal expression of Cav-1 in a cohort of DCIS patients treated with wide-excision and close follow-up and examined the progression to invasive breast cancer.

\section{Material and Methods}

DCIS breast tissues were obtained from the Surgical Pathology files at the Thomas Jefferson University. Seventy-eight cases for which histologic slides and blocks were available were included in the study. Clinical and treatment information was extracted by chart review. All patients were 列

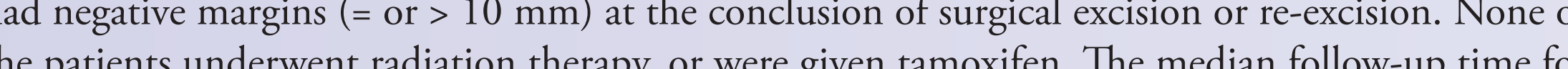
the patients underwent radiation therapy, or were given tamoxifen. The median follow-up time for papillary, micropapillary, comedo), nuclear grade, presence of necrosis and inflammation were

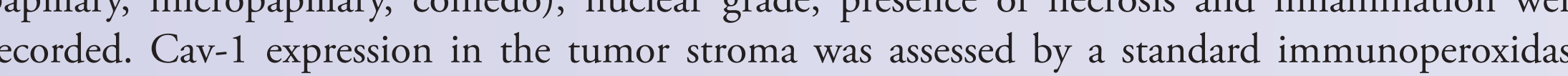
ethod (anti-caveolin-1 IgG, BD Biosciences,San Jose, CA; 1:50 dilution). The staining was scored semi-quantitatively as negative ( 0 ; no staining), weak ( 1 ; either diffuse weak staining or strong f the stromal cells). All hypothesis testing was performed using the Fisher exact test or the Kruskal-Wallis test, depending on the discrete or continuous nature of the other factors.

\section{Results}

Sixteen DCIS patients underwent some form of recurrence: 8 recurred to DCIS, while the other 8 recurred with progression to invasive breast cancer. Nearly ninety percent of DCIS patients $(7 / 8)$ that underwent recurrence to invasive breast cancer had reduced or absent levels of stromal Cav-1. Representative examples of Cav-1 immunostaining are shown in (Figure 1). The absence of stroma

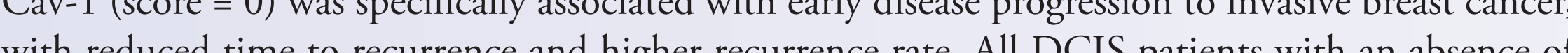
with Cov to invasive breast cancer (Figure 2). This represents an overall cumulative incidence of 100\% for recurrence and 80\% for progression. An absence of stromal Cav-1 in DCIS lesions was also specifically associated with the presence of inflammatory cells. Inflammation (the presence of lymphocytes and/or macrophages) was specifically associated with DCIS progression to invasive breast cancer (Figure 3) however this effect was strictly dependent on stromal Cav-1 levels Conversely, ninety-seven percent of DCIS patients with high levels of stromal Cav-1 (score $=2)$ did not show any invasive recurrence over the duration of follow-up (4-208 mo), and $89 \%$ of such patients are estimated to remain free of invasive recurrence, even after $15 \%$.

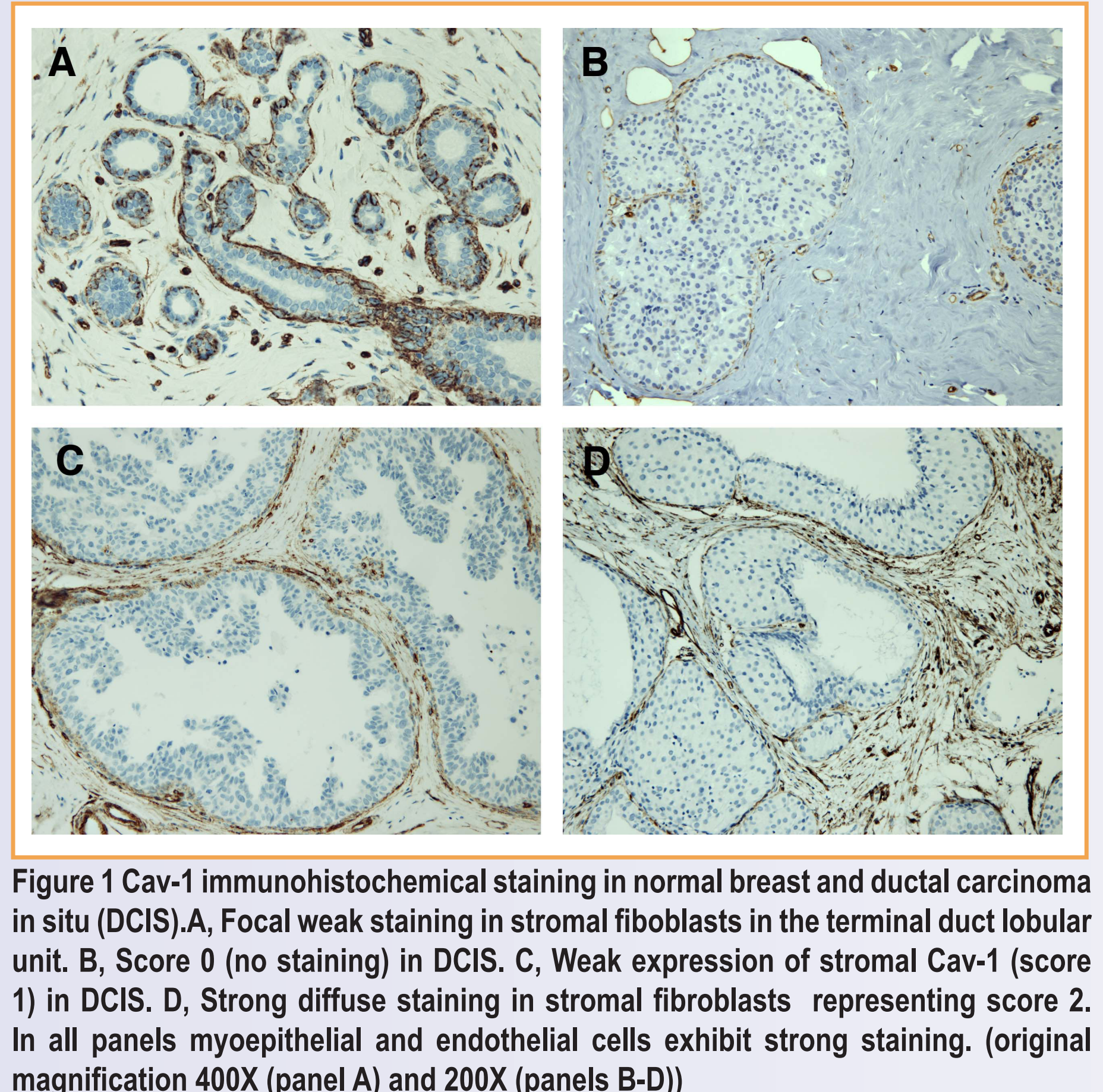

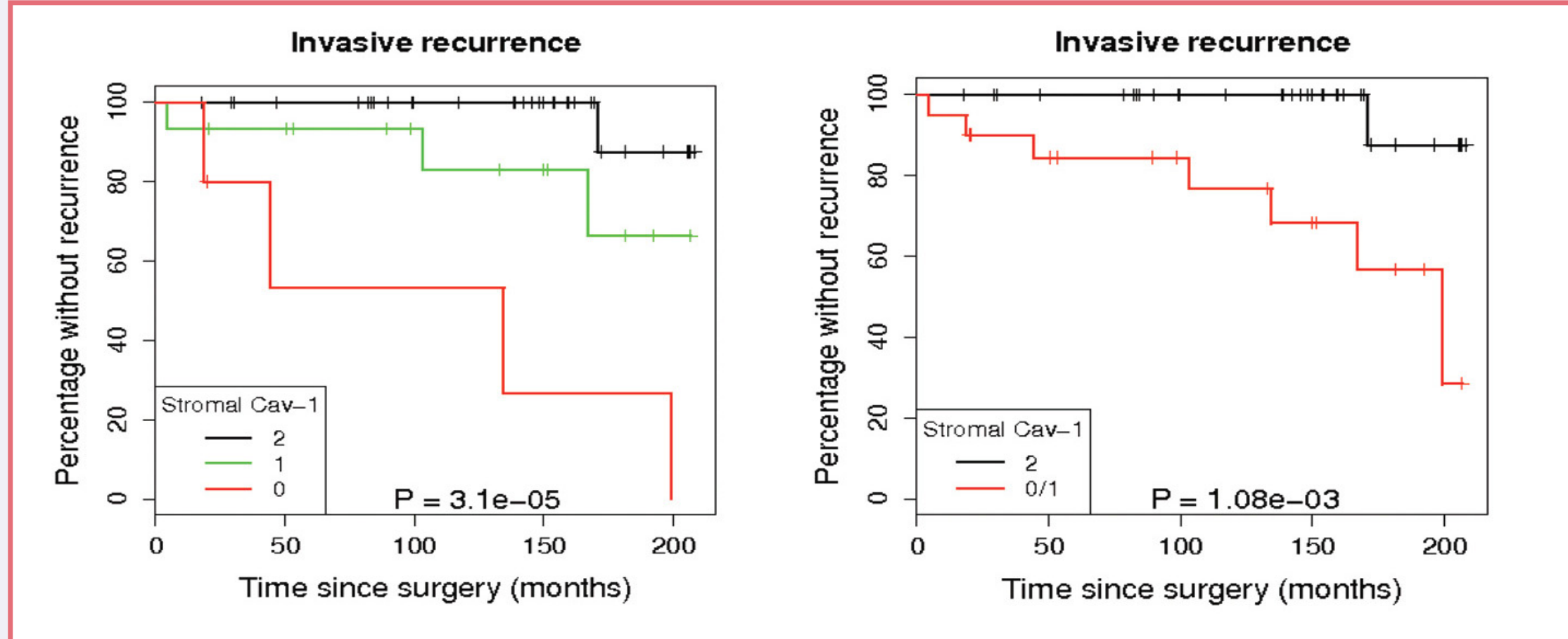

Figure 2. Kaplan-Meier curves for stromal Cav-1 status and time to overall recurrence among DCIS $\mathrm{pa}$ tients. Note that an absence or reduction of stromal Cav-1 is strongly associated with an increase progression to invasive breast cancer. On the left, patients were stratified into three different groups. On the right, patient
are as shown.

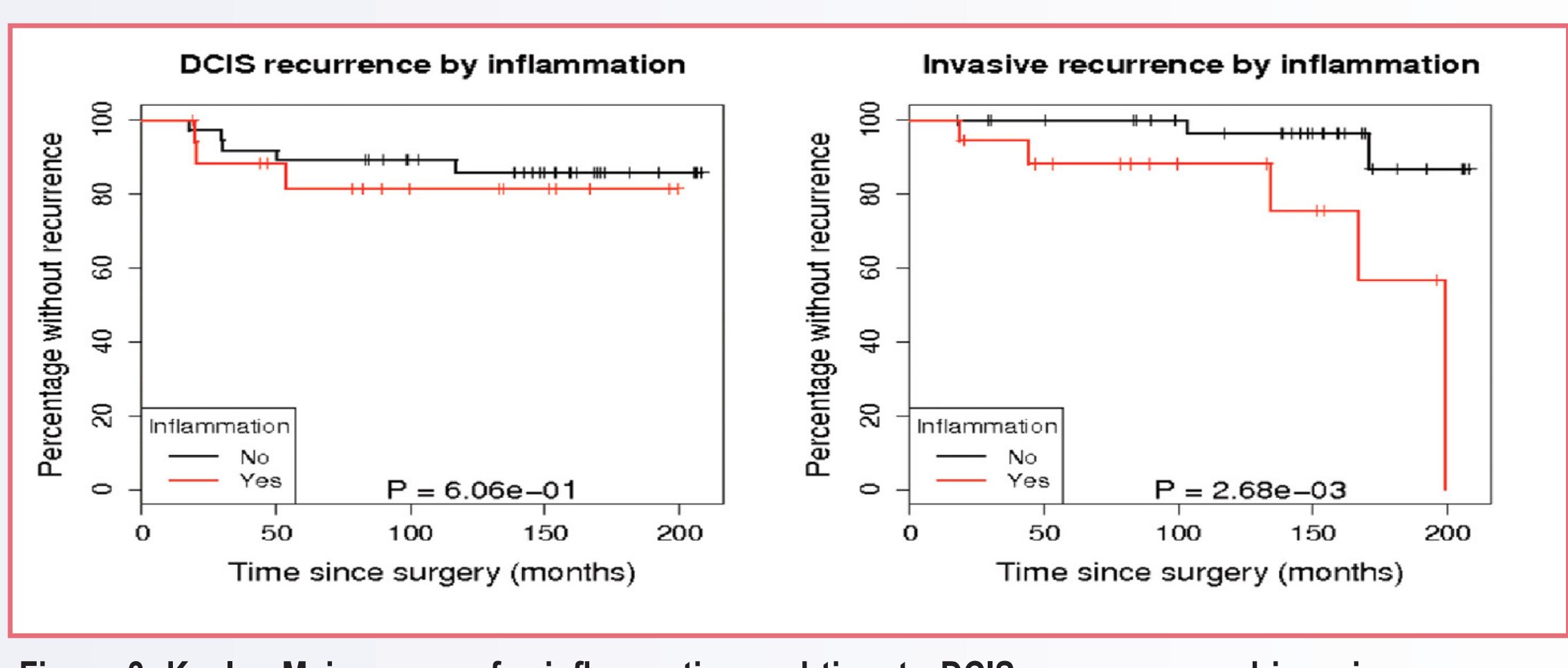

Figure 3. Kaplan-Meier curves for inflammation and time to DCIS recurrence and invasive recurren vasive breast cancer, but not simple DCIS recurrence, p values (log rank test) are as shown.

\section{Conclusions}

Stromal Cav-1 may represent a valuable new biomarker for dividing DCIS patients into high-risk and low-risk groups at diagnosis, facilitating their treatment stratification. 